\title{
Derivative Matrix Isopotential Synchronous Fluorescence Spectroscopy for the Direct Determination of 1-Hydroxypyrene as a Urinary Biomarker of Exposure to Polycyclic Aromatic Hydrocarbons
}

\author{
Yao-Qun LI, ${ }^{\dagger}$ Wei SuI, Chun Wu, and Li-Jun Yu \\ The Key Laboratory of Analytical Sciences of MOE and Department of Chemistry, Xiamen University, \\ Xiamen 361005, China
}

\begin{abstract}
Urinary 1-hydroxypyrene is a biomarker in the measurement of human exposure to polycyclic aromatic hydrocarbons. A rapid and simple derivative isopotential synchronous fluorescence method was developed for the direct determination of 1-hydroxypyrene in urine. A length of iso-intensity route was scanned on the three-dimensional fluorescence spectrum of urine and this result was combined with that from derivative technique. Thus the strong background signals of urine were removed and the 1-hydroxypyrene can be determined directly in urine without tedious pre-separation. The derivative isopotential synchronous fluorescence spectrum was directly obtained from a single scan on a spectrofluorometer, which further simplified isopotential synchronous fluorescence technique. The recoveries of $93 \%$ to $115 \%$ were obtained for 1 hydroxypyrene added to urine.
\end{abstract}

(Received October 2, 2000; Accepted October 28, 2000)

Polycyclic aromatic hydrocarbons (PAHs) widely present in the environment are produced by incomplete combustion or pyrolysis of organic materials, some PAHs are known to be carcinogenic. Human may be exposed to PAHs from a wide variety of occupational, environmental and dietary sources. An important approach in assessing human exposure and uptake of PAHs is to measure PAH metabolites excreted in urine. In particular, 1-hydroxypyrene, as a major pyrene metabolite in human urine, has been used as a biomarker in the measurement of human exposure to PAHs. ${ }^{1-6}$

Until now, chromatographic separation with fluorescence detection has been a major approach to determine urinary 1hydroxypyrene. Strickland et al. ${ }^{1}$ used high-performance liquid chromatography (HPLC) combined with constant-wavelength synchronous fluorescence detection, and gas chromatographymass spectrometry to measure pyrene-containing metabolites. A coupled-column HPLC method was suggested for the determination of 1-hydroxypyrene in urine. ${ }^{2}$ Solid phase extraction of pyrene metabolites followed by HPLC separation was developed to analyze 1-hydroxypyrene. ${ }^{3}$ Zhao et al. ${ }^{4}$ developed an analytical procedure to detect urinary 1hydroxypyrene by re-extracting it into an aqueous medium from organic phase, and subsequently measuring the constantwavelength synchronous fluorescence. Ramasamy et al. ${ }^{4}$ developed a solid matrix luminescence method to detect 1hydroxypyrene in HPLC urine fractions. Although the selectivity has been improved by the utility of conventional constant-wavelength synchronous fluorescence, the present methods of urinary 1-hydroxypyrene remain a time-consuming multi-step or expensive process.

Conventional constant-wavelength synchronous fluorescence

† To whom correspondence should be addressed.

E-mail: yqlig@xmu.edu.cn is limited to scan a $45^{\circ}$ cut on excitation and emission matrix. Several embranchments of synchronous fluorescence have been developed and wider and wider applications have resulted. ${ }^{7-13}$ Among them, matrix isopotential synchronous fluorescence (MISF) spectroscopy, which was pioneered by Pulgarin and Molina, ${ }^{7-9}$ is a novel technique for resolving highly-overlapped mixtures or detecting analytes in complex fluorescent background without the need for tedious prior separation. The technique involves performing synchronous scans through a trajectory that joins points of equal intensity on a fluorescent matrix three-dimensional spectrum. Since the MISF signals of background or interfering component are constant, they can further be compressed by the use of a first-order derivative technique.

In this paper, a rapid and simple method for the direct detection of urinary 1-hydroxypyrene in a single analysis is described by using derivative MISF techniques. This new method does not require either chromatographic separation or extraction procedures. Besides, we simplify MISF technique by obtaining MISF spectra directly from a single scan on a spectrofluorimeter instead of acquiring MISF spectra by postprocessing of three-dimensional fluorescence spectra.

\section{Experimental}

\section{Reagents}

1-Hydroxypyrene (98\%) was purchased from Aldrich and used as received. Sodium acetate and iced acetic acid were of analytical-reagent grade. $\beta$-Glucuronidase (110200 units/ml) was obtained from Sigma Chemicals. A buffer solution $(\mathrm{pH}$ 5.0) was prepared by mixing adequate amounts of $0.1 \mathrm{M}$ sodium acetate and $0.1 \mathrm{M}$ acetic acid. Deionized and distilled water was used. 


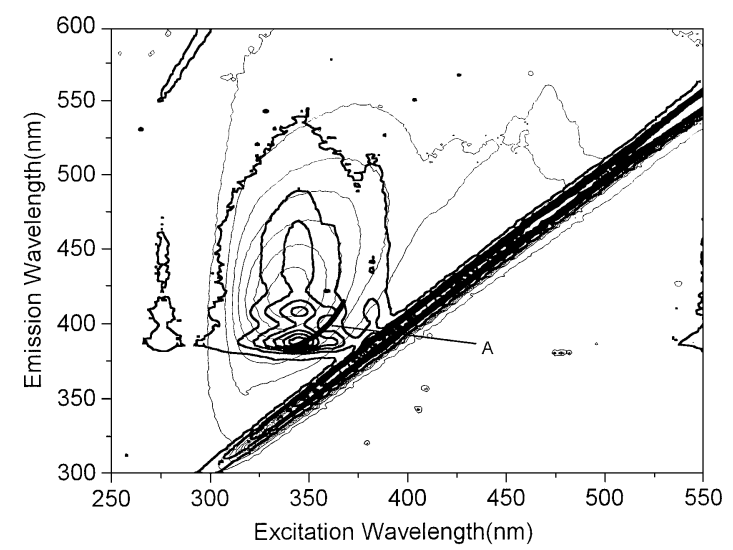

Fig. 1 Contour plots for 1-hydroxypyrene $(0.1 \mu \mathrm{g} / \mathrm{ml}$, bold line $)$ and urine sample (thin line). The bold line labeled as A in the plot is the scanning route for detection.

\section{Apparatus}

All spectra were obtained on a laboratory-constructed computer (IBM)-controlled spectrofluorometer that was similar to one previously described. ${ }^{12,13}$ The spectrofluorometer was equipped with a 500W Xenon lamp, the slit bandpasses of excitation and emission monochromators were set at $5 \mathrm{~nm}$. In addition to conventional fluorescence spectra, the apparatus could provide all kinds of synchronous spectra. We have modified the spectrofluorometer to make it suitable for the direct scanning of MISF spectra. An electronic differentiator was connected to the spectrofluorometer directly to obtain first- or second-derivative spectra. A quartz glass cuvette with a pathlength of $1 \times 1 \mathrm{~cm}$ was used throughout.

\section{Procedure}

Aliquots of $20 \mathrm{ml}$ fresh human unexposed urine samples were buffered with $10 \mathrm{ml}$ sodium acetate buffer ( $\mathrm{pH}$ 5.0) and hydrolyzed enzymatically with $0.02 \mathrm{ml} \beta$-glucuronidase for $1 \mathrm{~h}$ at $37^{\circ} \mathrm{C}$ in a water bath. The samples were then filtered and kept in a refrigerator.

The stock urine samples were diluted 10 times with buffer solution ( $\mathrm{pH}$ 5.0) before measurements. The three-dimensional fluorescence spectra of the diluted urine samples and $0.1 \mu \mathrm{g} / \mathrm{ml}$ 1-hydroxypyrene standard solution ( $\mathrm{pH}$ 5.0) were recorded as a stack of excitation spectra, varying the emission wavelength in steps of $2 \mathrm{~nm}$.

A suitable MISF scanning route was selected by trial-anderror based on the three-dimensional spectral information of urine and 1-hydroxypyrene. For the preparation of the calibration graph in urine, aliquots containing $0.05-10 \mu \mathrm{g} \mathrm{1-}$ hydroxypyrene were transferred into a $10 \mathrm{ml}$ calibrated flask, adding $1 \mathrm{ml}$ urine, diluted with sodium acetate buffer ( $\mathrm{pH}$ 5.0) to the mark. Combining with derivative technique, firstderivative MISF spectra were inquired directly from the spectrofluorometer. Peak-to-peak measurement was used for the detection of 1-hydroxypyrene.

\section{Results and Discussion}

\section{Spectral characteristics}

Urine samples contain many fluorescent components and produce strong fluorescence signals over a broad wavelength range. The fluorescence emitted from 1-hydroxypyrene was encompassed by that of urine, as seen from contour maps for 1-

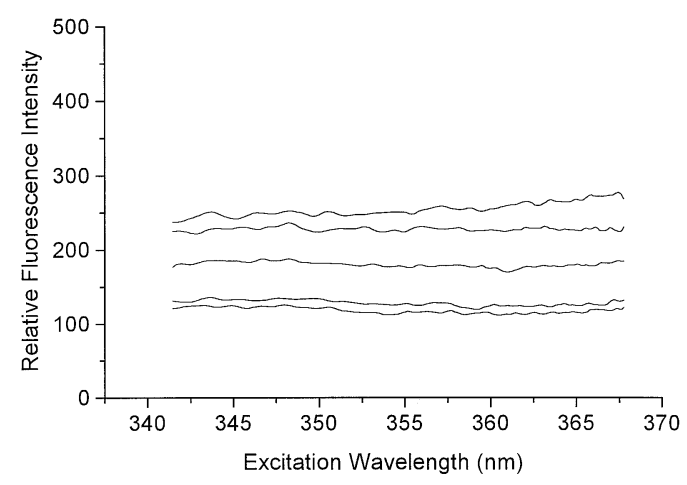

Fig. 2 MISF spectra for different urine samples.

hydroxypyrene and urine in Fig. 1. The serious spectral overlap of 1-hydroxypyrene and urine has made it impossible to resolve 1-hydroxypyrene in urine by means of direct conventional fluorescence approaches or even normal non-linear synchronous fluorometry. Previous works require either chromatographic separation or extraction procedures. Suitable chemometric analysis is possible, but time-consuming data acquisition of three-dimensional fluorescence spectra is required. MISF provides a simple method to detect selectively a fluorescent compound in the presence of a considerable unknown fluorescent overlapped background.

\section{Selection of MISF scanning route}

To find out a suitable scanning route in establishing a MISF approach is a key procedure. The scanning route of MISF is actually a trajectory of equal fluorescence intensity (contourline) on a total fluorescence spectrum of matrix background or interfering component. Firstly, the maximum of fluorescence intensity for 1-hydroxypyrene ( $\left.\lambda_{\mathrm{ex}} 348.5 \mathrm{~nm}, \lambda_{\mathrm{em}} 388 \mathrm{~nm}\right)$ was selected as a detection point. At this detection point, the fluorescence intensity of urine matrix was searched. A set of $\lambda_{\text {ex }}$ and $\lambda_{\text {em }}$ for the constant value of fluorescence intensity of urine was retrieved. Then a trajectory which joined the equal intensity points was defined as indicated in Fig. 1. After trial and error, a length of this constant-intensity trajectory (marked A in Fig. 1) formed the scanning route of MISF for the detection of 1-hydroxypyrene in urine.

Different urine samples were measured to test the applicability of the selected scanning route. As shown in the MISF spectra of five urine samples (Fig. 2), the MISF signals of each sample generally remained constant with wavelength in the scanning wavelength range, though the intensities were not the same for different samples. The results indicated that the scanning route is really a length of contour-line of urine, being independent of different samples. The concentrations of urinary components may differ with samples, which resulted in the fluctuation of fluorescence intensity. However, the composition ratios of urine samples were generally identical so that the shape of MISF spectra of urine was consistent. Some variance of MISF signals with wavelength might be observed in some other wavelength range, which indicated the composition of some components' contributing fluorescence in these ranges may vary from sample to sample. The MISF approach provides a flexible tool to select a suitable scanning route and to avoid those unstable wavelength ranges.

All MISF spectra reported in the literature ${ }^{7-9}$ have been obtained by post-processing of three-dimensional fluorescence spectra and the acquirement of three-dimensional data has been 


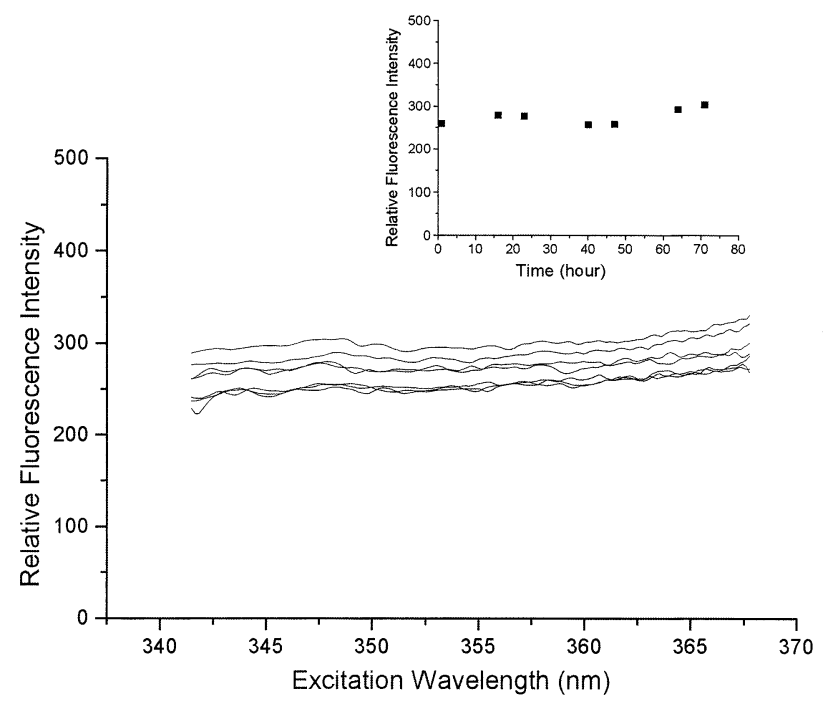

Fig. 3 MISF spectra of the same urine sample scanned within three days. The inside plot is the mean fluorescence intensity of the sample against time.

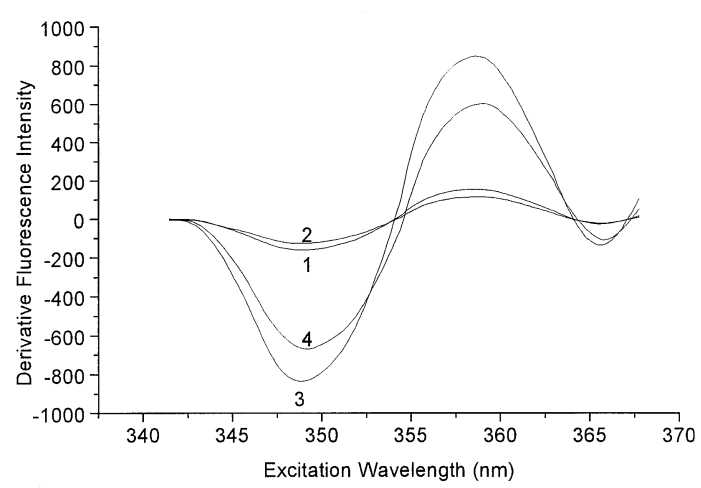

Fig. 4 First-derivative MISF spectra of $0.1 \mu \mathrm{g} / \mathrm{ml} 1$-hydroxypyrene in buffer solution (1) and in urine sample (2); and $0.5 \mu \mathrm{g} / \mathrm{ml} 1$ hydroxypyrene in buffer solution (3) and in urine sample (4).

a time-consuming work. In our method, we only used threedimensional fluorescence spectra to define a suitable scanning route. After finishing this work, it is unnecessary to acquire three-dimensional data again. The MISF spectra obtained in our work were directly scanned from the spectrofluorometer.

\section{Stability of urine samples}

Figure 3 is a series of MISF spectra of a urine sample which were scanned within three days. The inside plot was inserted to show the variance of the same sample as the average fluorescence intensities within the scanning wavelength range against time. The experimental results exhibited that the MISF signals were basically identical within selected scanning wavelength range, though the sample was detected at a different time, which demonstrated that the component composition in a urine sample was essentially stable with time. The difference of the intensities in each scan was partly attributed to the fluctuation of the experimental conditions.

\section{Influence of fluorescence quenching}

The fluorescence quenching effect of 1-hydroxypyrene by urine was apparent. 1-Hydroxypyrene in urine samples gave fluorescence signals $15-25 \%$ smaller than those in aqueous

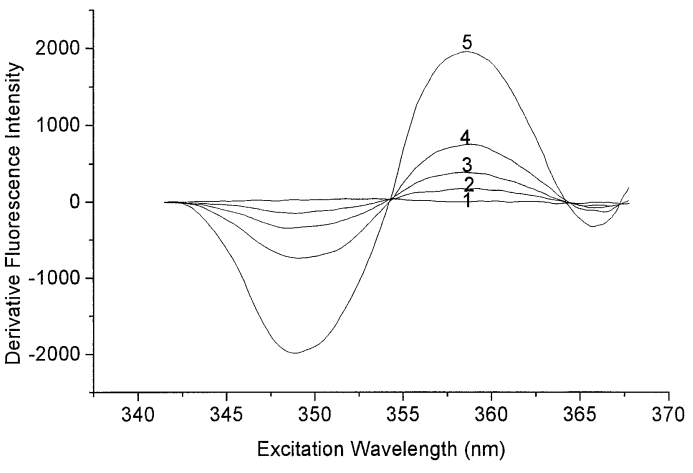

Fig. 5 First-derivative MISF spectra of 1-hydroxypyrene in buffer solution with concentrations of (1) 0 ; (2) 0.005 ; (3) 0.01 ; (4) 0.02 and (5) $0.05 \mu \mathrm{g} / \mathrm{ml}$.

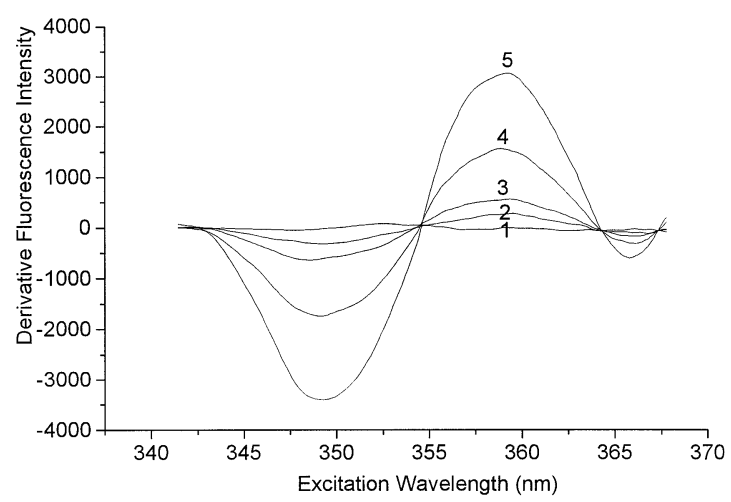

Fig. 6 First-derivative MISF spectra of 1-hydroxypyrene in urine sample with concentrations of (1) 0 ; (2) 0.01 ; (3) 0.02 ; (4) 0.05 and (5) $0.1 \mu \mathrm{g} / \mathrm{ml}$.

standard solutions (Fig. 4), which should be attributed to the complex of urine composition and the numerous absorption particles. Then, it is not suitable to construct calibration graphs of 1-hydroxypyrene in buffer solution for the propose of quantifying urinary 1-hydroxypyrene.

\section{Determination of 1-hydroxypyrene in urine}

Some derivative MISF spectra of 1-hydroxypyrene obtained in buffer solution and in urine was shown in Figs. 5 and 6 . Combining with first-derivative technique, we reduced the constant MISF values of blank urine to zero. This procedure was useful for the quantitative measurement of 1hydroxypyrene in urine. Though the constant MISF values of blank urine may differ with different samples, the firstderivative values of different blank urine samples should all be zero. Derivative signals of MISF for 1-hydroxypyrene were measured by peak-to-peak approach $\left(a^{\prime}-a\right)$. Calibration graphs of 1-hydroxypyrene were plotted as derivative signals against concentrations and prepared both in buffer solution and in urine. A statistical analysis of the experimental data by fitting the least-squares line according to $y=a+b x$ was carried out. The results are summarized in Tables 1 and 2. In Table 1, calibration series 1 and 2 were performed, respectively, in experimental sensitivity scale $\times 1$ and $\times 4$. Sensitivity $\times 1$ is in highest sensitivity and there are three-time relationships between adjacent sensitivity scales. In Table 2, calibration series 1,2 and 3 were performed, respectively, in experimental sensitivity scale $\times 1, \times 4$ and $\times 3$. For comparison, the data of original slopes $(b)$ were converted into those $\left(b^{*}\right)$ under the highest sensitivity scale 
Table 1 The results of calibration series in buffer solution applied to the least squares regression $(y=a+b x)$

\begin{tabular}{|c|c|c|c|c|c|}
\hline $\begin{array}{l}\text { Calibration } \\
\text { series } \\
\text { number }\end{array}$ & $\begin{array}{l}\text { Concentration of 1- } \\
\text { hydroxypyrene/ } \mu \mathrm{g} \mathrm{ml}^{-1}\end{array}$ & Intercept (a) & Slope (b) ${ }^{\mathrm{a}}$ & Slope $\left(b^{*}\right)^{b}$ & $\begin{array}{l}\text { Correlation } \\
\text { coefficient }(r)\end{array}$ \\
\hline 1 & $0.005-0.005$ & -91 & $8.07 \times 10^{4}$ & $8.07 \times 10^{4}$ & 0.9999 \\
\hline 2 & $0.05-1.0$ & 15 & $2.92 \times 10^{3}$ & $7.89 \times 10^{4}$ & 0.9998 \\
\hline
\end{tabular}

a. Measured in different experimental sensitivity scale.

b. Converted into highest sensitivity scale.

Table 2 The results of 1-hydroxypyrene calibration series in urine applied to the least squares regression $(y=a+b x)$

\begin{tabular}{|c|c|c|c|c|c|}
\hline $\begin{array}{l}\text { Urine } \\
\text { sample }\end{array}$ & 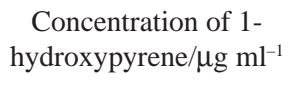 & Intercept (a) & Slope (b) ${ }^{\mathrm{a}}$ & Slope $\left(b^{*}\right)^{b}$ & $\begin{array}{l}\text { Correlation } \\
\text { coefficient }(r)\end{array}$ \\
\hline 1 & $0.005-0.1$ & -39 & $6.54 \times 10^{4}$ & $6.54 \times 10^{4}$ & 0.9998 \\
\hline 2 & $0.05-1.0$ & 10 & $2.42 \times 10^{3}$ & $6.53 \times 10^{4}$ & 0.9996 \\
\hline 3 & $0.01-0.5$ & -34 & $7.43 \times 10^{3}$ & $6.69 \times 10^{4}$ & 0.9997 \\
\hline
\end{tabular}

a. Measured in different experimental sensitivity scale.

b. Converted into highest sensitivity scale.

Table 3 The recoveries of 1-hydroxypyrene in urine

\begin{tabular}{cccc}
\hline $\begin{array}{c}\text { Urine } \\
\text { sample }\end{array}$ & $\begin{array}{c}\text { Amount added/ } \\
\mu \mathrm{g} \mathrm{ml}^{-1}\end{array}$ & $\begin{array}{c}\text { Amount found/ } \\
\mu \mathrm{g} \mathrm{ml}^{-1}\end{array}$ & Recovery, \% \\
\hline \multirow{3}{*}{$\mathrm{I}$} & 0.020 & 0.023 & 115 \\
& 0.040 & 0.042 & 105 \\
& 0.120 & 0.124 & 103 \\
$\mathrm{II}$ & 0.050 & 0.053 & 106 \\
& 0.080 & 0.083 & 104 \\
& 0.240 & 0.236 & 98 \\
$\mathrm{III}$ & 0.100 & 0.093 & 93 \\
& 0.200 & 0.192 & 96 \\
& 0.280 & 0.300 & 107 \\
\hline
\end{tabular}

(Tables 1 and 2). Calibration graphs of 1-hydroxypyrene in buffer solution and in urine samples were all linear over the concentration range $0.005-1.0 \mu \mathrm{g} / \mathrm{ml}$. The lower slopes of calibration graphs obtained in urine samples confirmed the quenching effect of urine matrix. Therefore, in order to measure urinary 1hydroxypyrene, a series for plotting calibration graphs should be prepared in urine. Calibration in real samples is a typical measure to reduce the matrix effect of real world. Consistent results among urine samples were obtained as exhibited by three calibration series in three urine samples respectively (Table 2).

Recovery experiments were performed in order to test the feasibility of the proposed method. Known amounts of 1hydroxypyrene were added to several different urine samples. The derivative signals of MISF were then measured. The found concentrations were read from the corresponding calibration graphs. The recoveries of 1-hydroxypyrene in urine samples are summarized in Table 3. The three urine samples used for the recovery test and those for calibration graphs were different. The calibration graph for urine sample 2 was utilized for measuring recoveries of 1-hydroxypyrene in the presence of urine samples I and II, and the calibration graph for urine sample 3 was utilized for measuring recoveries of 1 hydroxypyrene in the presence of sample III. The obtained recoveries of urinary 1-hydroxypyrene ranged from 93\% to $115 \%$.
To test the repeatability of the method, a urine sample containing $0.20 \mu \mathrm{g} / \mathrm{ml}$ 1-hydroxypyrene was measured 11 times and the relative standard deviation of $1.7 \%$ was obtained. The detection limit of $0.84 \mathrm{ng} / \mathrm{ml}$ for 1-hydroxypyrene in urine was obtained based on three times standard deviation of urine blank according to the IUPAC definition.

\section{Acknowledgements}

The supports by the National Natural Science Foundation of China and the Ministry of Education of China are gratefully acknowledged.

\section{References}

1. P. T. Strickland, D. Kang, E. D. Bowman, A. Fitzwilliam, T. E. Downing, N. Rothman, J. D. Groopman, and A. Weston, Carcinogenesis, 1994, 15, 483.

2. K.-S. Boos, J. Lintelmann, and A. Kettrup, $J$. Chromatography, 1992, 600, 189.

3. R. Singh, M. Tucek, K. Maxa, J. Tenglerova, and E. H. Weyand, Carcinogenesis, 1995, 16, 2909.

4. Z. Zhao, D. Tian, and W. Quan, Chin. Environ. Chem., 1994, 13, 141.

5. S. M. Ramasamy, R. J. Hurtubise, and A. Weston, Appl. Spectrosc., 1997, 51, 1377.

6. P. Strickland, D. Kang, and P. Sithisarankul, Environ. Health Perspect., 1996, 104 (sup.5), 927.

7. J. A. M. Pulgarin and A. A. Molina, Anal. Chim. Acta, 1994, 296, 87.

8. J. A. M. Pulgarin and A. A. Molina, Microchem. J., 1995, $52,341$.

9. J. A. M. Pulgarin, A. A. Molina, and P. F. Lopez, Analyst, 1997, 122, 247.

10. M. Hernandez Lopez, M. A. Gonzalex, and M. I. L. Molina, Talanta, 1999, 49, 679.

11. E. L. Inman and J. D. Winefordner, Anal. Chem., 1982, 54, 2018.

12. W. Sui, C. Wu, and Y. Q. Li, Fresenius J. Anal. Chem., in press.

13. Y. Q. Li, F. Qian, and X. Z. Huang, Chin. Chem. Lett., 2000, 11, 613 . 